\title{
A STUDY ON CONTRASTIVE PARALLEL-STRUCTURED SENTENCES UNDER SEMANTIC FIELD PERSPECTIVE
}

\section{Nguyen Van Thao}

Faculty of Philology, Hanoi Pedagogical University 2, Vinh Phuc, Vietnam

nguyenvanthao@hpuz.edu.vn

\begin{abstract}
Contrastive parallel-structured sentence (CPSS) was a traditionally artistic kind of Vietnamese literature, which was originated from the Chinese. The aims of this article is to investigate all kinds of the CPSS and the semantic field aspects inherently existing in it; adding that the article targets in what social occasions the Vietnamese use the CPSS the most. To have a deep insight into the semantic field aspects in the CPSS, the article has applied integrated methods such as descriptive method, analytical method and statistic method. In this article, the semantic field in the CPSS was showed, analyzed and discussed to reveal the application of linguistic units such as the phenomena of synonyms, antonyms, homonyms, fixed phrases and especially semantic field, adding that to show how words are exploited with the same semantic field.
\end{abstract}

Keywords: Nguyen Khuyen, CPSS, Chinese-based CPSS, semantic field.

\section{INTRODUCTION}

Contrastive parallel-structured sentence (CPSS) is a kind of Vietnamese traditional artistic literature originated from China. Its uniqueness is exclusively manifested by two semantically structured sentences. The first sentence is called the given proposition, and the second is modulated as the opposed proposition. Linguistic units employed in a CPSS are concise, condensed and highly suggestive in their content and form. Normally, the CPSS is composed by a person who delivers 'given proposition', and another person who opposes it with an 'opposed proposition'. However, in other cases, there is also a CPSS made by an individual only. One of the most typical and celebrated of such authors who had composed a great numbers of CPSS was Nguyen Khuyen. Who had composed CPSS on various occasions such as: celebrating success at exams, the inauguration of new houses, condolence expressions for the dead, wishing Vietnamese traditional Tet, etc. The author was very skillful in exploiting linguistic units such as the phenomena of synonyms, antonyms, homonyms, fixed phrases and especially semantic fields. In this article, the semantic fields indicating condolence in the CPSS will be 
investigated and analyzed to reveal the application of linguistic units such as the phenomena of synonyms, antonyms, homonyms, fixed phrases and especially semantic field, adding that to show how words are exploited with the same semantic field.

Up to now, there has been no deeply studied research on the semantic fields in Chinese-based CPSP, therefore, this article will further clarify the linguistic features under the semantic field theory.

\section{Researches on the CPSS}

Most works on the Chinese-based CPSS are from the perspectives of literary theory, stylistics, and historical literature. With reference to the types of CPSS, Tuyet T.T.N (1997) mentioned that

opposition rules and parrot rules in the CPSS were a unique literary form of the Vietnamese. The author referred to the principles or rules of CPSS including: 1 . The CPSS must have two propositions: 'given proposition' and 'opposed proposition'. It is not considered as a complete CPSS if it has only one or three propositions. Moreover, the two propositions of the CPSS must oppose each other in segments, syllables, tones, ideas, and lexicons; 2. Syllable is a mandatory linguistic unit in the CPSS. Interestingly, the CPSS should always have a number of syllables because its inherent propositions must be symmetrical and equally balanced in syllable. In the CPSS, the meaning of words in the opposed proposition is called the literal puzzle, and the opposite words of the two propositions must have the same parts of speech. Quyen. P.A (2010) on analyzing linguistic features in Nguyen Khuyen's Chinese-based literary works, the author mainly focused on the analysis of Chinese-based poetry. In regards to the CPSS, the author only collected the statistical data of paronomasia such as reduplicated words, the usage of functional words, and semantic aspects. However, Thanh. V (2003) in "Nguyen Khuyen - authors and works" merely compiled all the writings relating to Nguyen Khuyen's literature over the historical periods.

\section{Researches on the semantic field}

The development of semantic field theory was originally mentioned by the works of American anthropologists and German linguists in the early 20th century. These scholars were influenced by Humbold whose theory was about the inner speech-form of language. This theory reflects the unique cognitive ability of the world and the characteristics of each ethnic group and plays the role as the background for all theories of semantic field. However, Humboldt's theory had not been the only foundation for the formation of semantic field theory because its linguistic approach did not deviate from Saussure's structuralism (Guo, 2010). de Saussure (1973) stated: "the value of any elements are determined by surrounding factors". On the basis of this doctrine, some 
other researchers have taken various views on the semantic field, which include Porzig, Stern, Trier, Jolles, Ohman, Matoré, Ullmann, Oskaar, Buttler, Perchonock and Werner, Kleparski, Lehrer and some other researchers. However, Trier's semantic field theory opened a new era in the history of semantics (Kleparski and Rusinek, 2007:189). Later a series of authors such as Porzig and Ipsen, R. Hallig and W. Von Warburg, P.M Roget came up with different views on this issue. Besides, some specific studies on the semantic field can also be mentioned such as R. Hallig and W. Von Warburg (Chau, 1998:247), P.M. Roget (Chau, 1998:246) and W.P. Zaleskij who analyzed some verbs that indicate eye activity in English (Chau, 1998). Duchace studied the "conceptual field of beauty in modern French" (Chau, 1998:248). Some other authors such as Adrienne Lehrer (1985) studied the effect of the semantic field on the semantic change. Ricardo Mairal Usón (1990) in Spain studied the semantic field of light and darkness in the poem Paradise Lost by John Milton, and the study of Bert Peeters (1991). Since the 2000s, in many countries around the world, many studies have applied the semantic field's theory to study specific cases in one or several languages. In China: Guo. C (2010) applied the semantic fields theory to the teaching of English vocabulary for students; Chunming Gao and Bin Xu (2013) applied it to the study of English vocabulary, and (Weijie (2001) studied the sematic field in English. In Iran, Parvaneh Khosravizadeh and
Samira Mollaei (2011) studied auxiliary particle in the sense of the semantic field approach. In Oman, Ali Nasser Harb Mansouru (2012) studied translation errors in the semantic fields of English and Arabic. In Ukraine there is Anna Smoliana, PG (2013) and Jamil Qasim Hameed (2013) etc.

A number of Vietnamese authors have profited from the achievements of foreign authors to introduce semantic field theory in Vietnam. They include: Tu. N.V (1967), Chau. D.H (2007), Hung. D.V (2010), Khanh. N.T (1996), Toan. B.M (1999), (2012), (2014) and Tam. D.T.H (2011). In addition, a number of Masters theses studies the sematic field such as Thao. D.P (2010), Thoa. M.T.M (2012) etc.

\section{The Theories}

\subsection{The CPSS and Opposition rules}

\section{- The Opposition rules}

The opposition rules must artistically comply with certain rules, which is the basic principle in shaping the CPSS and the rule-based poetry in Tang Dynasty. Here, the significance is to find the balance among the opposite words or language units. In regard to phonetics, opposite words must have opposed tones égal and non-égal, and the opposite of rhythm structure. Moreover, parts of speech must be the same for opposite words such as noun noun, verb - verb, functional word - functional word, and pronoun - pronoun, etc. Similarly, in word-formation, it must be in a parallel formula: single words - single words, 
compound word - compound word and so forth. In phrases, the patterns are as follows: noun phrase - noun phrase, verb phrase - verb phrase, etc. The pair also includes consistent idioms, proverbs opposite each other; sentences with the same opposite pattern: simple sentence with simple sentence, simple sentence with auxiliary components opposite simple sentence with auxiliary components, etc. In ancient materials, there are a great number of the opposition rules namely chinh opposition rule, dich danh opposition rule, di loai opposition rule, lien chau opposition rule, song thanh opposition rule, diep van opposition rule, song nghi opposition rule, lien cam opposition rule and hoi van opposition rule ${ }^{1}$. To produce a complete CPSS, people frequently compete among the authors who must be smart and erudite (Sang, 2006). The opposition rules make use of isomorphic compound words. In literature, they are expressed in categories such as idioms, proverbs, and the rule-based poetry in Tang Dynasty including: two cau $d e^{2}$ (sentences 1 and 2 ) and the last two sentences without opposition rule; the cau thuc (sentences 3 and 4) are opposite to each other; and the two cau luan (sentences 5 and 6) are opposite to each other, too. The opposition rules also exist in van bien ngau ${ }^{3}$.

\footnotetext{
${ }^{1}$ They are different kinds of opposition rules.

${ }^{2}$ One kind of sentence in poetry

${ }^{3}$ Van bien ngau is one kind of literature
}

\section{- The CPSS}

Generally, when an individual comes up with a given proposition, he or she must be in charge of dealing with an opposed proposition in a certain situation. However, the individual may equally produce an entire CPSS. The CPSS is a special form of miniature literature with each unit of work (called a sentence) consists of two propositions (actually two sentences or two compulsory parts) which are symmetrical in terms of parts of speech, sound and meaning. They are used in expressing thoughts, feelings and attitudes towards a person, an event, a scene, or an object that the subject (author) is interested in. The CPSS can be employed with various purposes to display goodwill, an answer, congratulations, records, condolence expressions, or simply to make fun and laugh (Can. N.T, 1975). The above aspects are both the context and the purpose of the CPSS. The characteristics of the CPSS also lie in its newness, profound content and perfection, unique form, exclusive language with high generalization and concision shaping the power of CPSS.

Vietnamese CPSS can be divided into different categories with diverse rules determined on the author's implications. Nevertheless, the framework of a study does not allow us to present those divisions. 


\subsection{The semantic Field}

Each semantic system represented by a set of words is called a semantic field, in which words are semantically related or have the same semantic category. The semantic relations in a semantic field can be described" "we can generalize the semantic relationships between the semantic fields and the semantic relations in each semantic field" (Chau, 2007:170).

Thus, the semantic relationship between words must be considered in the system of the semantic field. The sememes in a semantic field have interrelated, intertwined and prescriptive relation. The constituent elements of a semantic field have common sememes (in addition to distinct sememes). Conversely, semantic relationships between semantic fields must be considered in the larger system of the semantic field. This confirms the systematic meaning of the languages.

According to Chau (2007:170), the semantic field was divided into two categories: horizontal and vertical semantic field. The vertical semantic field can be based on the meaning of words to distinguish the denotat and significate semantic fields. Furthermore, the associatively semantic field is established on the basis of characteristics of association.

\section{- Vertical semantic field \\ + Denotat semantic field}

A denotat semantic field is a set of words that are related to each other in terms of denotatum. The denotat semantic fields differ in the number of words, the arrangement of units and distribution domain in each language. When a word has many denotatum, it may belong to different semantic fields. The semantic fields can penetrate and interfere with one another when some words can be figured out in these semantic fields. The relation of words to each denotat semantic field is not the same. Some words are close-knit to the semantic field while some have very blur features to this semantic field. Based on the nature and relationship between the word and the semantic field, there are core characteristics that define the semantic field, including prototypes. As a result, the farther the gap between characteristics of words and the core characteristics is, the less the involvement in this semantic field is.

\section{+ Significate semantic field}

A Significate semantic field is a set of words that have the same significate structure. Moreover, a broad significate semantic field can also be divided into smaller significate semantic fields with different densities. Because a word has many significate meanings, they can belong to different significate semantic fields. The significate semantic fields interfere with and penetrate on each other, but the core characteristics are also preserved, especially in prototypes. To determine a significate semantic field, we base on a certain significate semantic structure as the root. 


\section{- Horizontal semantic field}

The horizontal semantic field first comes from the linearity of the linguistic signal; the signals take turn successively into a sequence that cannot occur simultaneously. Therefore this relationship is called horizontal relation or syntagmatic relation. To have a syntagmatic relationship with each other, the elements must perform the same function in terms of language or communication content. Thanks to the syntagmatic relationship, words expose their grammatical and lexical meanings. To create a linear semantic field, we choose a given word and then examine all words that have a similar relationship to the meaning of the root word and then combine them into acceptable linear sequences in a language.

\section{- Associative semantic field}

An associative semantic field is a set of words representing things, phenomena, activities, and properties, etc. which have an associative relation with the root word. To form an associative semantic field, the words first and foremost must belong to the horizontal and vertical semantic fields. Besides, words in an associative semantic field depend on the possible associative meanings of the given word. The associative semantic field also has many associative words which simultaneously appear with the root of word in relatively homogeneous and repetitive themes. Therefore, the associative semantic field has features of nationalism, age, and individuality.
They may have words with similar denotatum but also have different connotative meanings.

\section{METHODS}

This article used a qualitative approach. In addition, the case study was applied to analyze deeply the Nguyen Khuyen's condolence expressions at funerals under the semantic field perspective.

\section{Data Collection}

Nguyen Khuyen's CPSS as the primary data was collected by statistic and analytical methods from the following documents: Chau P (2008), Dieu X (1979), Giang M.L (1993), Hang N.B (2010), Huyen N.V (1984), Hiep H.S (1997), Sang T.L (2006), Thanh. V (2003) and Huy N.H (2003). There is a phenomenon in the same CPSS which some people attribute to Nguyen Khuyen while others believe it is from another authors. These cases are accepted and regarded as of Nguyen Khuyen. After subtracting the duplicated CPSS in the above constructions as well as the discarded variants, the number of remaining CPSS to be surveyed is 50. Subsequently, the 50 Chinese-based CPSS are divided into 5 categories in correspondence with the context of use. In the next step, the CPSS indicating condolence expression is investigated from the semantic field perspective. 


\section{Methods and Tactics}

2.1 Method of seme analysis

This method is employed to analyze words, groups of words belonging to semantic fields.

\subsection{Method of description}

This method is used to describe the results of the research.

\subsection{Tactics of statistics and classification}

These tactics determine the number of words belonging to context of use and to the semantic fields.

\section{FINDING S AND DISCUSSION}

\section{Findings}

On the basis of collecting and analyzing data, Nguyen Khuyen's Chinese-based CPSS are divided into five types in correspondence with their purposes: condolence, congratulation, traditional Tet holiday, teasing - sarcasm and commandment. At the same time, the phenomenon of using the semantic field in CPSS is also statistically treated and calculated among the different types as follows:

Table 1. Number and ratio of Chinese-based CPSS

\begin{tabular}{|c|c|c|c|c|c|c|c|}
\hline No & \multicolumn{3}{|c|}{ Types of Chinese-based CPSS } & Quantity & Rate & $\begin{array}{l}\text { Semantic } \\
\text { field }\end{array}$ & Rate \\
\hline 1 & \multicolumn{3}{|c|}{ Traditional Tet holiday } & 9 & 18 & 4 & 16 \\
\hline 2 & \multicolumn{3}{|c|}{ Condolence } & 16 & 32 & 10 & 40 \\
\hline \multirow{4}{*}{3} & \multirow{4}{*}{ Congratulation } & Longevity & 3 & \multirow{4}{*}{13} & \multirow{4}{*}{26} & \multirow{4}{*}{8} & \multirow{4}{*}{32} \\
\hline & & Wedding & 2 & & & & \\
\hline & & Housewarming & 2 & & & & \\
\hline & & Others & 6 & & & & \\
\hline 4 & \multicolumn{3}{|l|}{ Teasing - sarcasm } & 8 & 16 & 2 & 8 \\
\hline \multirow[t]{2}{*}{5} & \multicolumn{3}{|l|}{ Commandment } & 4 & 8 & 1 & 4 \\
\hline & \multicolumn{3}{|c|}{ Total } & 50 & 100 & 25 & 100 \\
\hline
\end{tabular}

The above result shows that most CPSS are composed to indicate condolence at funerals, which shows that they are closely related to the routinely life of the Vietnamese. It also shows his human outlook and worldview, even if the death but he doesn't completely avoid it. At the same time, it shows the culturally rooted activities through CPSS on occasions of death.
In terms of the quantity, CPSS has the largest number with $32 \%(16)$, the other high rate is for congratulatory with $26 \%(13)$, the remaining three types accounted for 18\% (9), $16 \%$ (8) and $8 \%$ (4) respectively. Thus the condolence expression is made the most of the 50 Chinese-based CPSS.

Regarding the number of CPSS using semantic field, condolence expressions have the highest proportion, accounted for $40 \%$ (10), the 
next highest rate are the congratulation with $32 \%$ (8), the following CPSS are traditional Tet holiday, teasing - sarcasm and commandment were $16 \%(4), 8 \%(2)$ and $4 \%(1)$ respectively.

This suggests that the employment of semantic field is quite common in Nguyen Khuyen's CPSS, especially condolence expressions in the funerals. The semantic fields used the CPSS indicating condolences are discussed in the following section.

\section{Discussion}

As mentioned above, Nguyen Khuyen's condolence expressions at funerals in the Chinese-based CPSS had 16 CPSS, accounting for $32 \%$ of the total. Among the 16 CPSS indicating condolence, the words of the same semantic field created different meaning layers. Compared to previous researches on the semantic field in general and the CPSS in particular, the semantic field features have been existed and broadly applied by the Vietnamese in such creative and sophisticated manners. Additionally, it can be reconfirming that the Vietnamese have taken advantage of a linguistic structure as the CPSS indicating condolence and congratulation to diversify their social lives. For example Semantic fields were mobilized to meet the purpose of addressing specific characteristics in describing the occupation of the dead as follows.

The dyer's wife cried because of her husband's death, which appeared a semantic field of various colors: biec (greenish), dark purple (tia), black, dieu (reddish), red, yellow, pink, white, purple (tim), blue. In other cases such as: The semantic field of butcher's tools and the semantic field for animal body parts in CPSS butcher's wife mourns her husband and children; The semantic field indicating the tools of a pig hog, an animal's body part, and the state of losing a body part in CPSS a wife of a hog who mourns her husband; In the blacksmith's wife mourns her husband there was a semantic field showing the tools and activities of the blacksmith; Chau van ${ }^{4}$ singer mourns her mother with semantic field showing musical instruments and how to play them and the sound of the music; In visiting the fathersin-law, there is a semantic field showing musical instruments; Guardians nun visiting monk has a semantic field that shows regency, items and activities; The widower mourns his wife uses a semantic field of the tools to confine and move chicken, the cry of the chicken and the situation of separation when having to separate; Finally, Crying wife has a semantic field of the typical dress of the farmer and the intellectuals.

Thus, Nguyen Khuyen employed quite a lot of semantic fields in his condolence expressions at the funerals, because in a CPSS with only from 4 syllables (or words) in each proposition to lower than one hundred syllables (or words), but the author has included opposing different semantic fields to create

\footnotetext{
${ }^{4}$ Chau van is one kind of Vietnamese song, recorded by UNESCO.
} 
meanings layers in his CPSS to express the feelings of the living with the dead.

\section{CONCLUSION}

Regarding Nguyen Khuyen's CPSS, there are $16(32 \%)$ condolence expressions, 13 (26\%) for congratulation, traditional Tet holiday gains $9(18 \%), 8(16 \%)$ for teasing - sarcasm and 4 (8\%) for commandment. The rate of making different types of Chinese-based CPSS shows that Nguyen Khuyen makes the most CPSS for condolence expression at funerals. Regarding the number of CPSS using semantic fields, condolence expressions of funeral has the highest proportion with $40 \%$ (10), next comes CPSS for congratulation with 8 cases, accounted for $32 \%$, the following cases are for traditional Tet holiday, teasing - sarcasm and commandment with $16 \%(4), 8 \%(2)$ and $4 \%(1)$ respectively. This suggests that the use of the semantic field is quite common in Nguyen Khuyen's Chinese-based CPSS, especially in the condolence expressions of funeral. Additionally, His CPSS also expresses the joys and sorrows of the people such as pain and empathy for the dead, New Year celebrations, housewarming, respect to the elderly etc. He was regarded as a man of the common people using a folk style of language to associate with the daily life of residents. Nguyen Khuyen's words are both academic and commonplace belonging to many semantic fields and conveying content closely and inextricably linked with the daily activities of the Vietnamese.

\section{REFERENCES}

Adrienne, L. (1985). The influence of semantic fields on semantic change. In: J. Fisiak (ed.), Historical Semantic, Historical Word formation. Berlin: Walter de Gruyter and co. pp.

283-296; http://alehrer.faculty.arizona.edu/sites/a lehrer.faculty.arizona.edu/files/Semantic \%20fields\%20and\%20change.pdf. (truy cập 25/2/2014).

Adrienne, L. and Eva, F. K. (1992), Frames, fields, and contrast (new essay in semantic and lexical organization), Lawrence Erlbaum Associates, Publishers Hillsdate, New Jersey Hove and London.

Ali, N. H. M. (2012) Semantic fields in English and Arabic: Problems in translation (được in trong cuốn Building Bridges: Integrating Languages, Linguistics, Literature, and Translation in Pedagogy and Research của Najma Al Zidjaly, Sultan Qaboos University, Oman, published by the Cambridge Scholars Publishing.

Bert P. (1991). A Few remarks on terminological insecurity in semantic field theory, Quaderni di semantic/ a. XII, n. 2, pp. 335-343.

Can. N.T. (1975). Vietnamese grammar (sound (syllable) - compound word - word phrase), $\mathrm{ÐH}$ and THCN, Hanoi, Vietnam.

Chau. D.H. (2007). Vietnamese Vocabulary Semantics, Hanoi: National University,

Chau. P. (2008). Vietnamese CPSS, Literature, Hanoi, Vietnam

Chunming G. and Bin X. (2013), The Application of Semantic Field Theory to English Vocabulary Learning, Theory and Practice in Language Studies, Vo.3, No. 11, pp. 
2035. Academy Publisher Manufacture in Finland.

Din. L.V. (2010), Semantics field of linguistic elements related to the water in folk songs, proverbs of Vietnamese, Language and Culture No. 9, Hanoi, Vietnam.

Dieu. X. (introduction) (1979), Poetry of Nguyen Khuyen, Hanoi Literature, Hanoi, Vietnam.

Giang. M.L (selection, introduction) (1993), Poetry of Nguyen Khuyen, Education, Hanoi, Vietnam.

Grzegorz A. K and Rusinek, A. (2007), The tradition of field theory and the study of lexical semantic change, Zeszyt, volume 47, pp. 187-205;

Guo. C. (2010). The application of the semantic field theory in college English vocabulary instruction, Chinese journal of applied linguistics, volume 33, no 3. pp. 50-62.

Hang. N.B (collect, compile). (2010), Vietnamese CPSS, Information Culture, Hanoi, Vietnam.

Hiep. H.S. (1997), Nguyen Khuyen, Van Nghe, HCM, Vietnam.

Hung. D.V. (2010), Some aspects of applying the semantic field in communication activities, Linguistics, issue 3 .

Huy. N.H. (2003), CPSS in Vietnamese Culture, Tong hop, HCM, Vietnam

Khanh. N.T. (1996), Characteristics of Semantic field of animal names (based on documents comparing Vietnamese with Russian) Thesis of Ph.D. in Literature, Institute of linguistics, Hanoi, Vietnam.

Jamil Q. H. (2013), Evaluation of the Semantic field theory and Componential Analysis as Theoretical Approaches of Potential Value to Vocabulary Acquisition: with Special
Reference to the Learner's Collocational Competence, Journal of the College of Arts. University of Basra, No 64.

Quyen. P.A. (2010), Literary characteristics of Nguyen Khuyen's Chinese-based poetry, master's thesis, Hanoi University of Education, Hanoi, Vietnam

Ricardo M. U. (1990), The Semantic Field of Light and Darkness in Paradise Lost. Sederi: Yearbook of the Spanish and Portuguese Society for English Renainssance Studies, no 1, pp. 189-208.

(Ricardo

Mairal

Usón, /www.sederi.org/docs/yearbooks / 01/1_13_mairal.pdf. (16/8/2013).

Sang. T.L. (2006), 5000 Hoang Phi Cau doi Han Nom. Information Culture, Hanoi, Vietnam.

Saussure, F. D. (1973), Linguistics, Social Sciences, Hanoi, Vietnam.

Tam. D.T.H. (2011), Semantic field of food and concept of HUMANITY, Linguistics, Issue. 5, Hanoi, Vietnam.

Thanh. V (selection and introduction) (2003), Nguyen Khuyen on author and works, Education, Hanoi, Vietnam.

Thao. D.P. (2010), The cognitive characteristics of Vietnamese people through the semantic fiel of "food", Master's Thesis, Hanoi University of Education, Hanoi, Vietnam.

Tuyet. T.T.N. (1997), Some remarks on the opposition rules and Parrot rules in Vietnamese CPSS, Linguistics, issue 2.

Thoa. M.T.M. (2012), Semantic field of Field in Vietnamese folk songs, Master thesis, Hanoi University of Education, Hanoi, Vietnam. 
Toan. B.M. (1999), Vietnamese Words in the use of communication, Education, Hanoi, Vietnam.

Toan. B.M. (2012), Fire in Truyen Kieu, Lexicography and Encyclopedia, issue 3, Hanoi, Vietam.

Toan. B.M. (2014), Words indicating the smell and taste in Truyen Kieu, Lexicography and Encyclopedia, issue 1, Hanoi, Vietam.
Tu. N.V. (1976), Modern Vietnamese words, $\mathrm{ÐH}$ \& THCN, Hanoi, Vietnam.

Weijie. Z. (2001), A New Research on English Semantic Field. Journal of Beijing International Studies University, 102, 3035. 